\title{
Erkrankungen der Nasennebenhöhlen und des Mittelohres bei Influenza.
}

Von Prof. Dr. Paul Prym,

Priv.-Doz. und I. Assistent am Pathologischen Institut der Universität in Bonn.

Während der letzten Influenzaepidemie konnte ich als Armeepathologe im Felde im Gebiete der Bugarmee von August bis Dezember 1918 über 100 Fälle von Grippe sezieren. Dabel habe ich besonderen Wert auf die Untersuchung der Keilbeinhöhle und des Mittelohrs gelegt. Da die Influenza wahrscheinlich mit einer Infektion der oberen Luftwege beginnt, ging ich von dem Gedanken aus, daß die Nebenhöhlen der Nase den Erregern vielleicht als Schlupfwinkel dienen würden, sodaß man hoffen konnte, dort den Influenzabazillus zu finden, der in der ersten Zeit, sonst nirgends nachgewiesen werden konnte. Damals handelte es sich ja noch um die Frage: Ist die ,Spanische Krankheit" eine echte Influenza? In der Tat gelang dieser Nachweis auch in einzelnen Fällen. Diese Befunde verloren aber an Wichtigkeit, als bekannt wurde, daß auch sonst der Nachweis von Influenzabazillen an anderen Stellen des Körpers und im Sputum häufig gelang. Ich setzte meine Untersuchungen aber fort, weil ich überraschend häufig schwere Veränderungen der Nebenhöhlen aufdecken konnte.

Aus dem Felde zurückgekehrt, sah ich dann, daß Fraenke ${ }^{1}$ ) sich mit denselben Fragen beschäftigt hatte, während sonst in der Literatur wenig darüber zu finden ist. Außer den bei Fraenkel erwähnten spärlichen Angaben aus der Literatur konnte ich nur noch Obduktionsbefunde von Weichselbaum²) aus dem Jahre 1890 feststellen. Weichselbaum sah in neun Fällen von Influenza stets eine Mitbeteiligung der Nebenhöhlen der Nase, besonders der Kieferhöhle, teilweise mit starker Eiterung.

Fraenkel dehnte seine Untersuchungen über sämtliche Nebenhöhlen der Nase aus, während ich vorwiegend die Keilbeinhöhle, gelegentlich auch die obere Nasenhöhle und die Stirnhöhlen berücksichtigte. Weiter dehnte ich dann meine Untersuchungen noch auf das knöcherne Gehörorgan und Mittelohr aus.

Im ganzen zeigen meine völlig von Fraenkel unabhängigen Untersuchungen, soweit sie die Nebenhöhlen der Nase betreffen, an einem noch größeren Material eine so weitgehende Uebereinstimmung, daß man die Resultate unserer Untersuchunger ruhig verallgemeinern kann und unsere Beobachtungen als konstanten Befund bei der Grippe ansehen muß

In 92 Fällen wurde die Keilbeinhöhle eröffnet. Mit breiten Meißel wird nach Entnahme der Hypophyse schichtweise paralle mit dem Klivus der Knochen abgemeißelt, bis die Schleimhaut der hinteren Wand der Keilbeinhöhle freiliegt. Es gelingt gewöhnlich ohne Verletzung der Schleimhaut, die knöcherne Höhle zu eröffnen. Man hat dann dell Vorteil, bequem von hinten her, aus der unverletzten Höhle Material zur bakteriologischen Untersuchung zu entnehmen. Immer wieder war ich erstaunt, wie verschieden weit die Keilbeinhöhle vom Klivus entfernt liegt; oft ist man beim ersten Meißelschlag an der Schleimhaut, oft muß man erst eine dicke Knochenschicht entfernen. Nach Entnahme des Materials wurde dann das Dach der Keilbeinhöhle auch noch entfernt, sodaß man einen völlig klaren Einblick hatte; von da aus läßt sich dann leicht die obere Nasenhöhle zugänglich machen.

Nur in 21 (von 92) Fällen wurde die Keilbeinhöhle völlig intakt gefunden. In 71 Fällen fanden sich Verärderungen von leichter Rötung bis zu völliger Vereiterung. In etwa $77 \%$ der Grippefälle fand sich also eine Veränderung der Keilbeinhöhle. Das stimmt fast genau mit den Befunden von Fraenkel an den Nasennebenhöhlen überein; die Keilbeinhöhle war bei Fraenkel allerdings nur in $58 \%$ der Fälle verändert. Berücksichtigt man aber, daß sich in zehn Fällen bei meinem Material nur eine leichte Injektion oder Rötung der Schleimhaut fand, die vielleicht auf der Grenze des Pathologischen steht; dann nähern sich auch hier meine Resultate den Fraenkelschen.

Auch in meinen Fällen handelte es sich überwiegend um exsudative Vorgänge. In über der Hälfte der Fälle fand sịch Eiter oder schleimig-eitriger Inhalt in der Keilbeinhöhle. Der Eiter wär meist gelblich, graugelblich, mitunter etwas grünlich. Auch die serösen Ergüsse waren meist gelblich, nur in vier Fällen bräunlich, gẹlbbrạ̈unlich oder graubräunlich, während Fraeṇkẹ viè häufiger hämorrhagischen Charakter der Ergüsse feststellte: Dem entsprachen auch meine Befunde an der Schleimhaut. Gewöhnlich war die Schleimhaut gerötet, oft gleichzeitig mehr oder weniger stark geschwollen, zweimal von fleckigen Blutungen durchsetzt; 6 mal ödematös geschwollen, 3 mal dabei ohne Rötung; mehrfach war die Schleimhaut blaß, die Höhle aber mit Eiter gefüllt. Nur einmal fand sich bei starker Rötung die Höhle leer. 
In 47 Fällon sandte ich Material zur bakteriologischen Untersuchung in das Laboratorium des Beratenden Hygiẹnikers ein. Die Untersuchung wurde in dankenswerter Weise unter Verantwortung von Herrn Dr. Prcygode ausgeführt.

Das Material wurde in 2-48 Stunden nach dem Tode entnommen; irgendeinen Einfluß auf die Resultate schien die frühere oder spätere Entnahme nicht zu haben; nur fiel mir auf, daß zum Schluß der Epidemie die Resultate häufiger negativ waren

In 25 Fällen ergab die Untersuchung ein positives Resultat, in 22 Fällen ein negatives.

8 mal wurden Pneumokokken gefunden $(1 \mathrm{mal}$ mit Gram-negativen Stäbchen),

4 mal Gram-positive Diplokokken,

6 mal Staphylokokken.

4 mal Influenzabazillen,

$1 \mathrm{mal}$ Influenzabazillen-ähnliche Stäbchen,

1 mal Streptokokken,

1 mal kein eindeutiges Resultat.

Die bakteriologischen Befunde lassen sich mit denen Fraenkels niclit vergleichen, da es natürich ein großer Unterschied ist, ob das Material im eigenen Institut in der Heimat mit allen Mitteln untersucht wird, oder ob es von anderer Seite im Felde nebenbei verarbeitet werden muß.

Bei positivem Influenzabazillenbefund fand sich dreimal schleimig-eitriger Inhalt in der Keilbeinhöhle, einmal mehr wäßriger, bräunlicher Inhalt. Außerdem fiel auf, daß in allen ubrigen positiven Făllen das Exsudat etwa zur Hälfte wäßrig, zur Hälfte eitrig war.

Auch in meinen Fällen waren die Erkrankungen im Leben nicht aufgefallen; die - allerdings oft sehr wenig ausführlichen Krankengeschichten erwähnen außer Kopfschmerzen keine Symptome, die auf Nebenhöhlenerkrankungen hinwiesen.

Der Tod erfolgte, soweit es sich aus den Krankengeschichten ersehen ließ

$$
\begin{aligned}
& \text { am 1.-5. Krankheitstag } 9 \mathrm{mal} \\
& \text { "6.-10. } \quad 81 \mathrm{mal}^{,} \\
& \text {"11.-15. } \quad \text { " } 15 \mathrm{mal} \\
& \text { "16.-20. } \quad 7 \mathrm{mal} \\
& \text { nach dem } 30 . \quad \text { " } \quad 1 \mathrm{mal},
\end{aligned}
$$

in den übrigen Fällen blieb die Krankheitsdauer unklar. Bestimmte Beziehungen der Krankheitsdauer zur Erkrankung der Keilbeinhöhle ließen sich nicht feststellen. Schon in den ersten Krankheitstagen fanden sich deutliche Veränderungen; beginnende Eiterung schon am vierten Tage; ebenso wurden mitunter die Keilbeinhöhlen am 17. bis 18. Krankheitstage völlig intakt gefunden. Das stimmt ganz mit den Beobachtungen Fraenkels überein.

Die obere Nasenhöhle wurde $48 \mathrm{mal}$ untersucht; etwa in der Hälfte der Fälle $(25 \mathrm{mal})$ fanden sich Veränderungen, von Rötung der Schleimhaut bis Eiterung; stets korrespondierend mit den Veränderungen der Keilbeinhöhle.

Die Stirnhöhlen wurden 33 mal untersucht; in etwa einem Drittel der Fälle $(10 \mathrm{mal})$ fanden sich ähnliche Veränderungen wie in der Keilbeinhöhle, im ganzen mit diesen korrespondierend.

Auf die Frage, ob die geschilderten Erkrankungen der Nasennebenhöhlen nicht zufällige Befunde darstellen, die garnichts mit der Grippe zu tun haben, brauche ich nicht näher einzugehen, da Fraenkel diese Frage ausführlicher behandelt hat. Zweifellos hängen diese Veränderungen mit der Grippe zusammen; entweder sind sie direkt durch die Erreger der Grippe hervorgerufen, oder das Grundleiden hat die Ansiedlung anderer Bakterien begünstigt.

Man kann dię Entzündung der Keilbeinhöhle wegen ihrer Häufigkeit bei Grippe für die Diagnose bei der Obduktion verwerten. Aus früheren Untersuchungen Fraenkels wissen wir, daß eine derartig häufige Mitbeteiligung der Nasennebenhöhlen bei keiner anderen akuten Infektionskrankheit gefunden wird. Ueber die klinisch so häufige Beteiligung des Mittelohres scheinen auch keine ausgedehnteren Untersuchungen bei der Obduktion gemacht worden zu sein.

Ich habe in 88 Fällen das knöcherne Gehörorgan und Mittelohr untersucht und $28 \mathrm{mal}$ Veränderungen gefunden. 2 mal handelte es sich um eine chronische Mittelohrentzündung; $26 \mathrm{mal}$ um akute Veränderungen, von leichter Rötung des Trommelfells und der Schleimhaut bis zur Vereiterung. $15 \mathrm{mal}$ war die Erkrankung einseitig, $11 \mathrm{mal}$ doppelseitig. 19 mal fand sich Eiter, davon 5 mal auch in den Warzenzellen; $4 \mathrm{mal}$ seröses Exsudat, davon $1 \mathrm{mal}$ ausschließlich in den Warzenzellen; 3 mal nur eine Rötung oder Injektion der Schleimhaut bzw. des Trommelfells. Hämorrhagisches Exsudat wurde nicht beobachtet.

Im ganzen korrespondieren die Befunde am Mittelohr mit denen an der Keilbeinhöhle: nur dreimal fand sich bei akuter Entzündung des Mittelohrs die Kèilbeinhöhle unverändert.

Die für Influenza klinisch so charakteristische Myringitis bullosa haemorrhagica sahen wir in keinem Falle bei der Obduktion.

Bakteriologisch wurde der Mittelohreiter nur in wenigen Fällen untersucht, das Resultat entsprach gewöhnlich dem Befunde in der Keilbeinhöhle; dreimal fanden sich Gram-positive Diplokokken, einmal Streptokokken, mehrmals war das Ergebnis negativ.
Kann man nun diese Otitiden als zum Bilde der Grippe gehörig ansprechen? Bakteriologisch haben wir dafür keinen Anhalt; der bakteriologische Befund spricht aber auch nicht dagegen, da nur wenige Fälle untersucht wurden. Die klinischen Befunde sprechen jedenfalls dafür, daß die Otitis media eine häufige Komplikation der Influenza ist. Es ist aber zu berücksichtigen, daß auch bei anderen Infektionskrankheiten eine häufige Beteiligung des Mittelohres gefunden wird, sodaß man die Otitis media bei der Obduktion diagnostisch nur mit Vorsicht verwerten kann.

Klinisch war die Otitis media mehrfach erkannt, meist war sie aber bei dem schweren Krankheitsbild übersehen worden

In allen obduzierten Fällen wurden schwere Lungenveränderungen nachgewiesen, über die ich mit den sonstigen Befunden an anderer Stelle berichten werde.

Abgeschlossen April 1919. 